\title{
Des énergies renouvelables pour l'Allemagne : Planification spatiale et technique pour une transition énergétique intelligente
}

Renewable Energy in Germany: Spatial and technical planning for an intelligent power supply

Erneuerbare Energie für Deutschland: Räumliche und technische Planung für eine intelligente Energieversorgung

\section{Stephan Bosch}

\section{OpenEdition}

\section{Journals}

Electronic version

URL: http://journals.openedition.org/rge/5412

DOI: $10.4000 /$ rge.5412

ISSN: 2108-6478

\section{Publisher}

Association des géographes de l'Est

\section{Printed version}

Date of publication: 1 January 2015

ISSN: 0035-3213

\section{Electronic reference}

Stephan Bosch, « Des énergies renouvelables pour l'Allemagne: Planification spatiale et technique pour une transition énergétique intelligente », Revue Géographique de l'Est [Online], vol. 55 / n $1-2$ | 2015, Online since 25 June 2015, connection on 21 December 2020. URL : http:// journals.openedition.org/rge/5412 ; DOI : https://doi.org/10.4000/rge.5412

This text was automatically generated on 21 December 2020.

Tous droits réservés 


\section{Des énergies renouvelables pour l'Allemagne : Planification spatiale et technique pour une transition énergétique intelligente}

Renewable Energy in Germany: Spatial and technical planning for an intelligent power supply

Erneuerbare Energie für Deutschland: Räumliche und technische Planung für eine intelligente Energieversorgung

Stephan Bosch

\section{Introduction}

1 L'objet de cet article est de montrer l'importance de la ressource que constitue l'espace pour la transformation de notre système énergétique en remplaçant les énergies fossiles et nucléaires par les énergies renouvelables. L'accent est mis d'abord sur les limites de la planification du développement des installations éoliennes et solaires de plein champ. Puis on présente différents concepts permettant de réaliser un réseau efficace de systèmes de production décentralisés indépendants des conditions météorologiques et qui peuvent contribuer à stabiliser les réseaux électriques. Dans la première partie de l'article, l'espace de recherche est le Land de Bavière qui se caractérise par un changement complet de paradigme au profit de l'énergie éolienne. Dans la deuxième partie, la perspective est élargie à l'ensemble de l'Allemagne afin de montrer les grandes disparités régionales auxquelles sont confrontés les protagonistes de la transition énergétique. 


\section{Problématique : énergies renouvelables et surfaces dans l'espace rural}

2 Le potentiel de croissance économique des zones rurales est généralement considéré comme faible (Henkel G., 2004, p. 222). Les avantages de situation tels que de grandes surfaces et les faibles prix du foncier auraient perdu leur ancienne importance et ne sauraient en aucun cas être suffisants pour attirer des flux d'investissements vers la périphérie. Cependant, on néglige le fait qu'avec la libéralisation des marchés de l'électricité en 1996, et en particulier avec l'introduction de la loi sur les énergies renouvelable de 2000 , la situation a considérablement changé car la production d'électricité décentralisée à partir du photovoltaïque, de l'énergie éolienne et de la biomasse requière des ressources spatiales considérables. C'est pourquoi on parle d' «energy from space», système dans lequel la production d'énergie nécessite beaucoup plus de surface qu'un système énergétique conventionnel (Brücher W., 2008, p. 4). Au total, la production d'un gigawatt - heure d'énergie finale nécessite 4,4 ha au moyen du photovoltaïque au sol, 5,7 ha pour des éoliennes et même 102 hectares avec des installations de biogaz (Peters J., 2010, p. 17). Les champs pétrolifères productifs, par contre, atteignent des rendements à l'hectare moyens de 10,000 à 40,000 t (Wikipedia, 2012). La densité énergétique des renouvelables est donc relativement faible. Cependant, la disposition décentralisée des systèmes de production des énergies renouvelables permet de réduire beaucoup les pertes en ligne entre la production et la consommation de courant, d'autant plus que l'on a plus à effectuer des changements entre autant de niveaux de tension (Klagge B. / Brocke T., 2013, p. 12).

3 Celui-ci doit être distingué d'un système énergétique centralisé, composé de quelques grandes centrales électriques qui n'alimentent pas le réseau électrique local, mais plutôt de puissants réseaux de transport nationaux. L'impact spatial d'un système énergétique fossile ou nucléaire est très faible lors de la production d'électricité. Les gisements ont des densités énergétiques élevées, mais ils sont inégalement répartis dans l'espace (Rempel H., 2008). Les coûts spatiaux n'apparaissent qu'avec la distribution mondiale de pétrole ou de charbon ; ce qui conduit à parler d' «energy for space» (Brücher W., 2008, p. 4). Les transitions entre les systèmes d'énergie décentralisée et centralisée sont certes progressives (Schmitt T., 2012, p. 253), car la construction de centrales solaires thermiques en Afrique du Nord pour l'alimentation d'une partie des centres de consommation européens, va avoir un fort impact spatial, par le biais de lignes de transmission à haute tension en courant continu et ce, aussi bien pour la production que pour la distribution de l'énergie (Walter K. / Bosch S., 2013).

4 En tout cas, l'utilisation prioritaire garantie par la loi sur les énergies renouvelables en Allemagne fait que désormais, des facteurs quantitatifs considérés jusqu'alors comme insignifiants tels que la disponibilité de terres et des bas prix du foncier, sont réévalués et offrent un avantage de localisation à des communes particulièrement fragiles. Le caractère décentralisé des technologies des renouvelables offre la possibilité de construire des structures d'approvisionnement autonomes et de stimuler des systèmes économiques régionaux (Bund Naturschutz, 2010). Les systèmes d'alimentation en énergie doivent être non seulement installés, mais aussi exploités et entretenus. Cela conduit à des contrats lucratifs pour les entreprises, les techniciens de maintenance et les fournisseurs de matières premières de la région. 
Toutefois, cet avantage économique pour les zones rurales ne peut pas compenser les bouleversements sociaux et environnementaux d'un développement surdimensionné des énergies renouvelables. La transition énergétique a augmenté de façon spectaculaire la pression sur les zones rurales et ainsi conduit à la prise de conscience que les utilisations énergétiques ne peuvent pas toujours équilibrer les coûts en termes spatiaux. Le problème est que la plus grande partie de l'expansion en Allemagne est encore à venir. À la fin de l'année 2012 les énergies renouvelables ne couvraient que 23 $\%$ de la consommation d'électricité (BDEW, 2012) et $12 \%$ de la consommation d'énergie primaire (cf. Figure 1). Une transition énergétique complète semble être encore bien lointaine. On est du moins parvenu à diminuer l'importance des sources d'énergie conventionnelles, à faire progresser la décentralisation de l'approvisionnement énergétique et à rompre le déjà long oligopole existant en matière d'offre. Pour continuer dans cette voie, il est nécessaire, dans les prochaines années, de poursuivre l'intégration spatiale des énergies renouvelables avec une plus grande sensibilité que ce n'était le cas auparavant. Ceci peut être réalisé par exemple par une expansion spatiale acceptable, en adaptant le choix de la technologie et son importance quantitative aux conditions spatiales locales (Bosch S., 2012). Si l'on n'y parvient pas, ce sont les conflits entre les nombreux acteurs locaux en attente de demandes d'utilisation des terres, qui s'aggraveront. La bonne image originelle des «technologies vertes» pourrait ainsi subir d'autres dégradations. Pour éviter cela, il faut d'abord avoir une idée plus précise des dimensions spatiales de l'expansion des énergies renouvelables (Bosch S., 2013).

Figure 1 : parts des différentes sources dans la consommation d'énergie primaire en Allemagne en 2012 (Bosch d'après Peyke et al. 2013, p. 49)

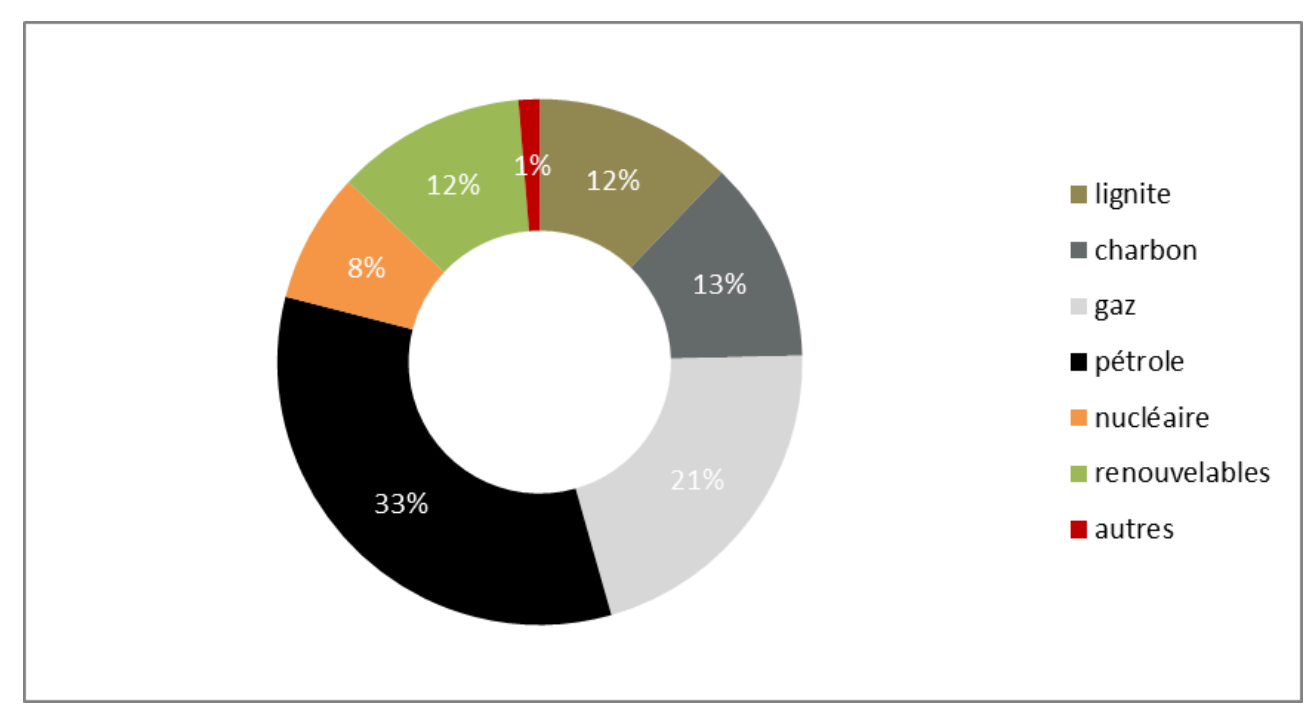


Figure 2 : importance de l'énergie nucléaire en Europe (Bosch) : carte représentant les réacteurs nucléaires en fonctionnement, en construction et prévus, ainsi que la part du nucléaire dans la production d'électricité du pays.

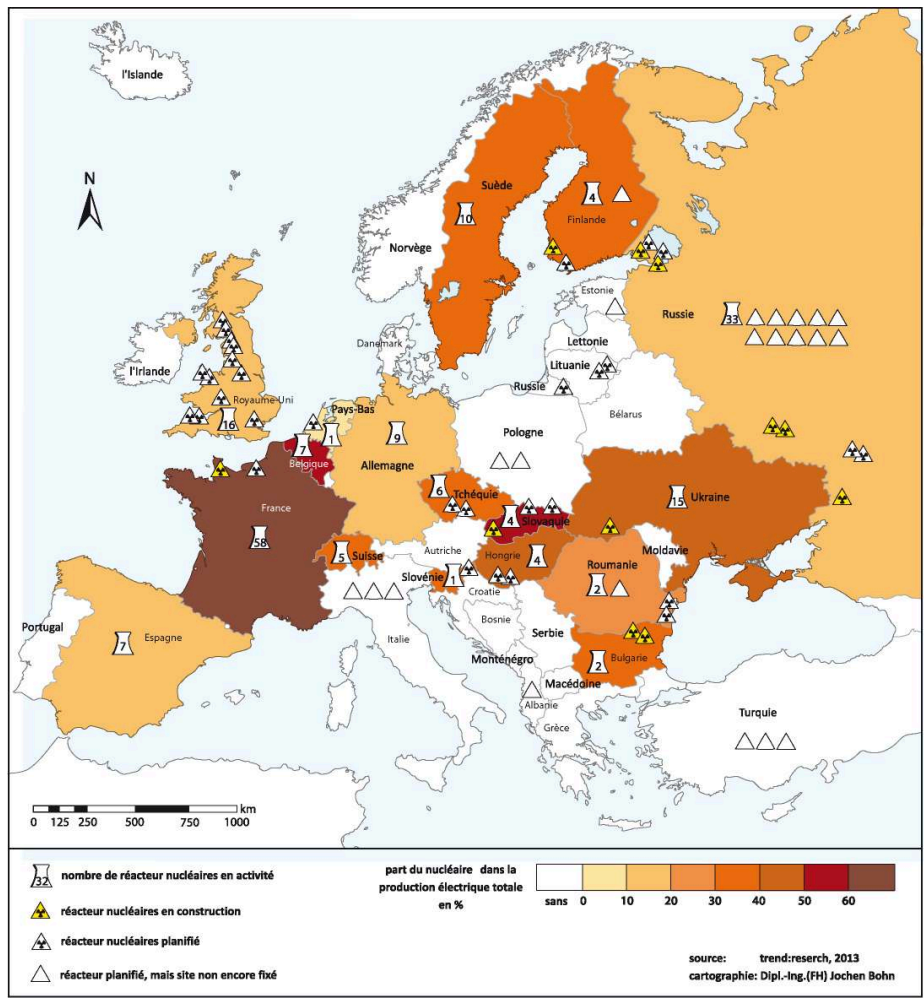

\section{Le nouveau départ d'une politique énergétique : la Bavière découvre l'énergie éolienne}

\section{A. Un grand potentiel spatial}

Depuis la décision surprise du gouvernement fédéral de fermer, au plus tard en 2022, les neuf centrales nucléaires encore en activité en Allemagne (WNA, 2013) (fig. 2), la Bavière qui est à plus de $50 \%$ dépendante de l'énergie nucléaire, s'efforce de promouvoir l'énergie éolienne terrestre jusqu'alors encore marginale (Weinhold N., 2012). Une étude menée par l'Institut Fraunhofer pour l'énergie éolienne et les technologies des systèmes énergétiques (IWES) a calculé un potentiel théorique de $17.000 \mathrm{~km}^{2}$ pour l'énergie éolienne en Bavière (IWES, 2011). En plus des surfaces sans restriction de principe, les zones forestières et les espaces protégés ont été inclus. Le problème avec cette approche de l' IWES est qu'elle ne prend pas en compte la gamme complète des options technologiques, mais privilégie l'expansion accélérée de l'énergie éolienne. Ce n'est donc pas un concept d'expansion adapté à l'espace acceptable, qui intègre les technologies des renouvelables dans le contexte régional, mais d'une technologie donnée qui est imposée sur différents sous-espaces. Il en résulte que les associations de protection de la nature et du patrimoine vont prendre position contre une telle étude unilatérale, puisque même avant Fukushima, la transformation excessive du paysage culturel par l'éolien industriel a été vivement critiquée (Bosch S. / Peyke G., 2011; Nohl W., 2001 / 2010). L'industrie éolienne soutient l'approche de l'étude IWES car l'énergie éolienne occupe encore trop peu de place en Bavière. À cet 
égard, les services de planification régionale ne manquent pas d'être critiqués car en dépit des objectifs ambitieux d'expansion du gouvernement fédéral, ils accordent trop peu d'espace à l'énergie éolienne. Les surfaces qui se prêtent à la construction d'éoliennes devraient donc être retirées des zones de protection des paysages et l'énergie éolienne devrait être inscrite comme un objectif dans la loi sur l'aménagement du territoire. Il faudrait au moins deux pour cent de la surface du Land de Bavière pour produire 80 TWh d'énergie éolienne par an (Zimmermann J.-R., 2011a). L'Etat libre de Bavière qui, avec 570 éoliennes et une capacité totale de $868 \mathrm{MW}$ (BWE, 2013), ne couvre qu'un peu plus d'un pour cent de ses besoins en électricité, vise d'ici à 2021 à porter la part de l'énergie éolienne à au moins 10\%. Mais pour cela, les surfaces consacrées à l'énergie éolienne devraient être multipliée par cinq (Einig K. et al., 2011). Selon le décret sur l'énergie éolienne, cela conduit à l'installation d'un maximum de 1.500 éoliennes supplémentaires. Pour y parvenir, il est nécessaire d'amener les services régionaux d'aménagement du territoire, plutôt réticents, à délimiter des zones prioritaires et des objectifs pour l'énergie éolienne (STMi et al., 2011).

\section{B. Les obligations de l'aménagement du territoire}

7 Une étude de l'Institut fédéral de la construction, de l'Urbanisme et de l'aménagement du territoire (BBSR, 2008) a pu confirmer L'hypothèse selon laquelle les besoins en surfaces du secteur de l'énergie éolienne sont insuffisamment pris en compte par les services de planification régionale de la Bavière. Avec l'aide du moniteur de planification spatiale (ROPLAMO, une base de données qui couvre toutes les zones d'aménagement pour l'énergie éolienne), les scientifiques ont pu montrer qu'au début de l'année 2011, 40 des 113 régions de planification en Allemagne ne prévoyaient aucune zone pour le développement de l'énergie éolienne. Dans les plans régionaux, le développement de l'éolien est prévu dans près de 2.000 zones, représentant une superficie totale de 131679 ha (0,37\% de l'Allemagne). Les disparités régionales sont cependant importantes et montrent les différences d'attitudes existant vis-à-vis de la technologie de l'énergie éolienne. Ainsi, au Schleswig- Holstein, où la capacité totale installée était de de $3.271 \mathrm{MW}$ en 2011, on a pu autoriser $0,87 \%$ de la surface pour l'énergie éolienne. En Bavière, c'était seulement 0,05\% (Einig K. et al., 2011; Weinhold N., 2012). Cet écart n'est pas simplement dû au fait que les conditions naturelles pour l'énergie éolienne en Bavière sont plus mauvaises qu'au Schleswig- Holstein, car la dernière génération d'aérogénérateurs assure également la rentabilité des installations éoliennes sur des sites de Bavière.

8 Ce qui explique les disparités spatiales considérables encore existantes, ce sont plutôt les préférences spécifiques à chaque Land en ce qui concerne l'orientation technologique pour la production d'énergie renouvelable. Jusqu'à présent, la Bavière est restée centrée sur les technologies du photovoltaïque et du biogaz, alors que l'option de l'énergie éolienne a été négligée (Bosch S. / Peyke G., 2009, p. 45). Dans une perspective spatiale, cela s'exprime par le fait que jusqu'en 2011, seulement sept des dix-huit régions d'aménagement bavaroises ont mis en place des règlements pour l'utilisation de l'énergie éolienne (Einig K. et al., 2011). Par conséquent, on peut en déduire qu'il existe des potentiels considérables de surfaces pour l'énergie éolienne dont la valorisation devrait être encouragée par les services de planification régionale. Toutefois, pour la délimitation des nouvelles zones prioritaires, les services de planification régionale devraient veiller à ce que l'exploitation des éoliennes existantes 
ne soit pas perturbée par de nouveaux parcs éoliens. Avec la construction d'éoliennes de plus en plus grandes et la réduction de la distance entre les zones, il pourrait en résulter des problèmes croissants de ralentissement $\mathrm{du}$ flux éolien et un développement de fortes turbulences dans la direction des vents dominants. Cet "effet de sillage" entre des éoliennes voisines conduit non seulement à une diminution du rendement pouvant atteindre 30\%, mais il est susceptible d'accroître les coûts de réparation et de maintenance. Dans ces conditions, la délimitation de nouvelles zones peut être contreproductive et doit être réalisée dans le prolongement des parcs déjà existants; sinon les surfaces consacrée à l'éolien vont s'accroître de manière disproportionnée. C'est ce qui explique qu'en raison du risque de se retrouver à l'abri d'un des parcs éoliens existant, les développeurs vont désormais s'assurer des zones adjacentes à leurs propres installations (Zimmermann J.-R., 2011b, p. 40).

En dehors de la définition de nouveaux sites potentiels, les services régionaux de planification vont également avoir la responsabilité de délimiter des zones d'exclusion pour l'énergie éolienne. En Bavière, il s'agit des parcs nationaux, des réserves naturelles, des zones centrales des réserves de biosphère, de secteurs de paysages protégés, de certains monuments naturels, des zones européennes de protection des oiseaux, ainsi que de la zone $\mathrm{C}$ du plan alpin. En principe, il est possible de construire des éoliennes dans les zones Natura 2000. Toutefois, les objectifs de protection de ce type d'espaces ne doivent pas être transgressés. Il existe également une sensibilité particulière dans les zones tampons des réserves de biosphère, les aires de protection des paysages, les forêts composées de vieilles futaies, où d'espèces d'arbres proches de l'état naturel, ainsi que dans les zones A et B du plan alpin. En outre, il est important de préserver l'esthétique de certains paysages patrimoniaux et de ce fait même touristiques, d'un trop grand impact technique (STMi et al., 2011). Une réduction future de la pression exercée par une expansion surdimensionnée de l'énergie éolienne sur les espaces ruraux découlera du fait que la consommation d'espace nécessaire pour produire une certaine quantité d'énergie aura tendance à diminuer. Cela est dû d'une part aux progrès techniques à l'origine de systèmes de production de plus en plus puissants qui vont remplacer les éoliennes moins puissantes (repowering). D'autre part, il est prévu que les responsables de la planification régionale prêtent davantage attention aux sites les plus productifs pour la désignation des zones prioritaires et en assurent la mise à disposition. En conséquence, le BBR table sur une réduction moyenne des besoins fonciers de l'énergie éolienne de 7 ha/ MW à 4,84 ha / MW (Einig K. et al., 2011). 
Photo 1 : parc photovoltaïque à Egglhausen en Bavière (photo : Klaus Leidorf)

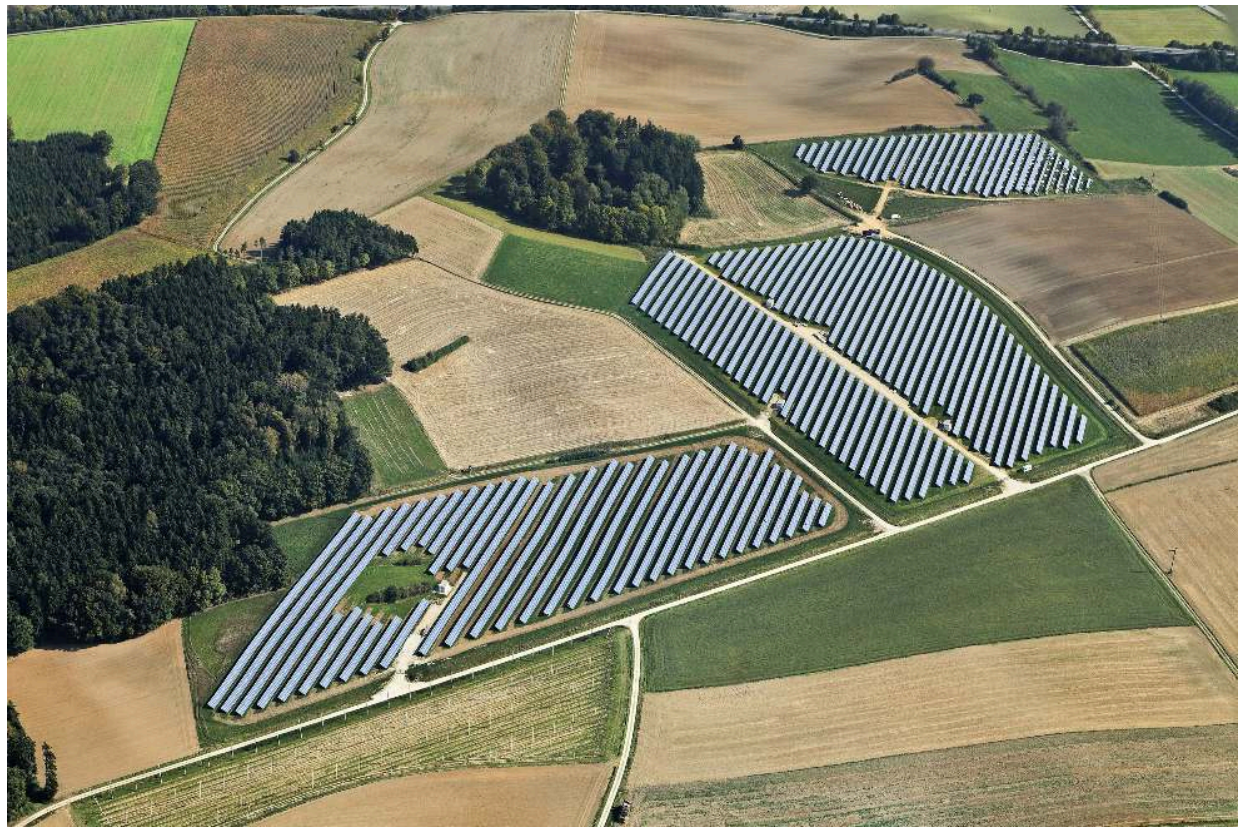

\section{L'esthétique paysagère}

Les discussions sur la dichotomie apparente entre le paysage culturel et les énergies renouvelables ont à leur tour contribué à un grand scepticisme quant à la production d'énergie renouvelable et ouvrent ainsi de nouvelles perspectives dans la recherche sur le paysage (Gailing L. / Leibenath M., 2013). Les éoliennes dont les dimensions sont généralement à l'origine d'une dégradation sévère des paysages se trouvent au centre de la critique (Nohl W., 2001). Cependant, il y a aussi des partisans de ce choix technologique. On n'en parle pas moins, pour l'expansion de l'énergie éolienne en Allemagne du Nord, de "miracle de l'énergie éolienne" ou de "success story" (Boeing N., 2011, p. 30). Cependant, le discours pro-éolien unilatéral est critiqué, d'autant plus qu'il est surtout tenu par les partis représentés au Parlement et donc plus fortement présents dans le débat public que les nombreuses voix critiques qui mettent en garde contre la transformation du paysage culturel par des éoliennes de plus en plus grandes (Leibenath M. / Otto A. 2012, p. 128). L'expression souvent utilisée de "défiguration du paysage culturel" (Brücher W., 2008, p. 10; Nohl W., 2010, p. 4), est cependant trompeuse car elle donne l'impression que les éoliennes sont partout dans l'espace et rendent impossibles des usages alternatifs. En effet, les éoliennes se présentent actuellement sous la forme de grandes installations particulièrement visibles et de ce fait, elles changent les caractéristiques esthétiques des espaces d'implantation, bien que les exigences spatiales de l'industrie éolienne soient le plus souvent rejetées par l'aménagement du territoire, et même pas du tout prises en compte dans les plans d'aménagement (Bosch S . / Peyke G., 2011c). La défiguration du paysage n'est donc pas réalisée par la loi d'aménagement du territoire ; ce reproche pouvant être interprété comme un témoignage du caractère destructeur de la culture de la discussion existant entre les partisans et les adversaires de l'énergie éolienne.

Il convient de noter que le rejet des éoliennes est généralement basée sur l'esthétique du paysage (Hasse J., 1999, p. 261). Cela n'est pas surprenant, parce que jusqu'à présent, 
la planification n'a pas du tout tenu compte des aspects esthétiques des paysages pour la détermination des règles d'utilisation et n'a donc pas respecté les statuts de restauration ou de recomposition des espaces. Par le biais de ce que l'on peut appeler la planification négative qui attribue à l'énergie éoliennes les seules zones sans restrictions et sans prérequis (donc des aires résiduelles), il ne peut pas y avoir d'adaptation de la technologie au paysage dans lequel elle doit être intégrée (Schöbel S., 2012, p. 21). Du point de vue de l'architecture du paysage, il est clair que l'énergie éolienne ne peut être ressentie comme étant esthétique que si l'on réussit à établir une relation nature - culture bien visible. Dans le contexte de la culture de la planification en cours en Allemagne, cela apparaît difficile, car une éolienne est jusqu'à présent considérée comme un mal inévitable, et non comme un nouvel élément signifiant du paysage, qui peut également constituer un apport culturel précieux à un paysage au caractère historiquement évolué. Il est en tout cas douteux que la planification régionale, qui distingue entre des espaces banals et des espaces de qualité, puisse par ses réglementations, répondre à un développement spatial équilibré (BMJ, 2008; Schöbel S., 2012, p. 22). On doit ici poser la question de savoir si les gens habitant dans des régions prioritaires pour l'éolien ont aussi droit à un beau paysage. Si tel est le cas, alors le problème de la défiguration du paysage doit être réglé par une disposition des éoliennes adaptée au paysage.

Les aspects esthétiques du paysage sont pris en compte jusqu'à présent dans le processus de planification à travers des indemnités compensatoires dont le montant dépend de l'importance de l'impact sur le paysage (Zimmermann J.-R., 2012, p. 25). La détermination du degré de défiguration va dépendre de la qualité du paysage affecté, de la situation topographique et de la hauteur des éoliennes (Knies J., 2010). Par exemple, pour la construction d'une éolienne de $200 \mathrm{~m}$ de hauteur et de $3 \mathrm{MW}$ de puissance dans une zone de paysage de haute qualité, on a fixé un paiement compensatoire de $240.000 €$. Ces paiements seront versés à des associations locales de protection de la nature, qui peuvent ainsi promouvoir leurs propres projets. L'aspect critique du montant de la compensation est qu'elle est en mesure d'empêcher certains des projets éoliens initialement rentables car de nombreuses zones paysagères de Bavière sont classées comme étant de haute qualité. De plus, cette obligation de compensation financière pour une mesure qui est essentiellement considérée comme écologique apparait irritante (Schöbel S., 2012, p. 19).

13 Par ailleurs, il faut aussi noter qu'il est pour le moins difficile de quantifier l'impact esthétique des éoliennes sur les paysages (Karl F., 2006). Le langage juridique parle très rarement d'une défiguration de l'image du paysage. Seul un paysage culturel méritant d'être strictement protégé et qui serait fortement dévalué par la construction d'une éolienne peut être affecté par le risque de défiguration. Le simple argument d'une dépréciation de l'image du paysage en raison de l'impact visuel (cette accusation pourrait s'appliquer à n'importe quelle infrastructure) n'est pas suffisant pour empêcher une implantation en dehors des zones préférentielles. Même la nécessité d'une accoutumance visuelle qui, au fond, concerne tout nouvelle technologie avec des matériaux en rupture avec le paysage, n'est pas juridiquement applicable (Scheidler A., 2010, p. 527). 


\section{La minimisation des conflits d'usage de l'espace}

Bien que depuis la réforme du code de l'urbanisme en 1997, un plan directeur n'est plus nécessaire pour l'approbation des éoliennes, les municipalités peuvent orienter le développement de l'énergie éolienne par la création de zones de concentration dans le plan d'occupation des sols. Ces zones sont déterminées dans le cadre de processus de concertation internes à la commune, ainsi que par la présentation d'un schéma de planification cohérent. Cette procédure est utilisée principalement pour offrir aux communes l'opportunité de participer à la conception de la transition énergétique. De plus, on peut ainsi mieux intégrer les particularités locales dans la planification et éviter un développement désordonné dans les zones non réservées par les communes. Cependant, le Tribunal administratif fédéral a longtemps renoncé à fixer un nombre minimum d'éoliennes à réaliser dans une zone de concentration. Par conséquent, certaines communes ont défini de très petites zones afin de ne pas susciter l'intérêt des développeurs potentiels de projets éoliens (Marque C. / Pohlmann K., 2010). Il convient de souligner que la délimitation de zones de concentration s'est accompagnée d'une exclusion de l'énergie éolienne dans le reste du territoire communal. Cette tactique a fortement réduit la probabilité que des investisseurs de l'industrie éolienne puissent encore s'intéresser à ces communes. La décision prise par la Bavière, dans le cadre du décret sur l'éolien, de ne plus lier la délimitation des zones prioritaires à une exclusion dans les zones extérieures atténue cet inconvénient au profit de l'industrie éolienne (STMi et al., 2011).

Figure 3 : évolution de la production d'électricité à partir de sources d'énergie renouvelable en GWh (Bosch, d'après Peyke et al. 2013, p. 19)

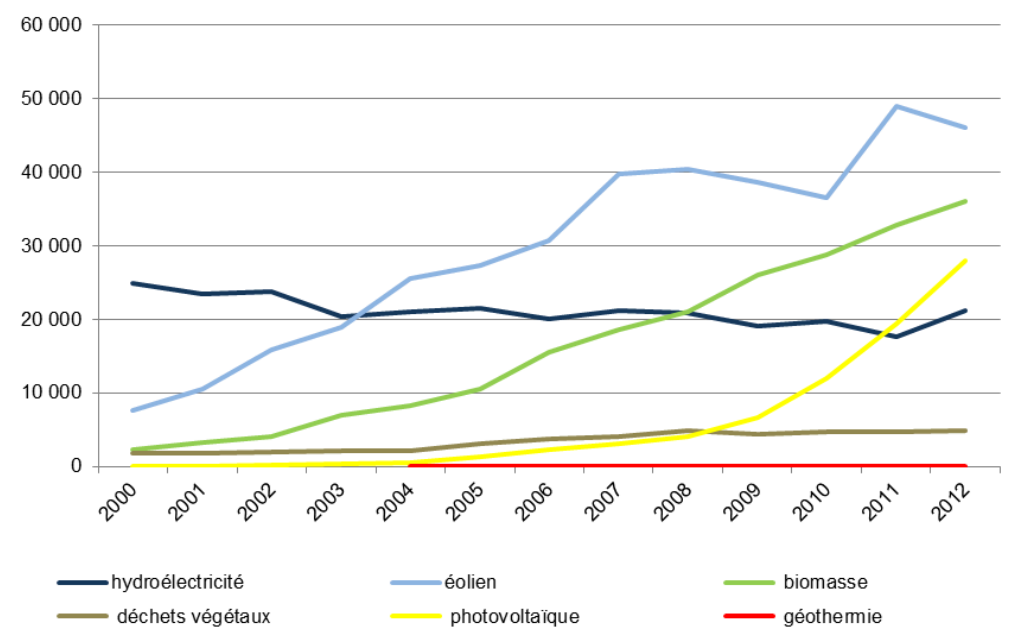




\section{La nouvelle orientation de la branche photovoltaïque}

\section{A. La césure spatiale de la loi EEG sur les énergies renouvelables de 2010}

Contrairement à l'énergie éolienne, le photovoltaïque n'est pas concerné par les dispositions prioritaires de l'article $\S 35$ de la section 1 du Code de l'urbanisme, qui permet une construction dans les espaces hors zones si elle n'est pas contraire à l'intérêt public comme par exemple la protection des monuments, la gestion du paysage ou la protection des sols (Code de l'urbanisme, 2012). Si un parc solaire doit être installé comme un système distinct à l'extérieur, un règlement municipal de zonage est nécessaire. Il n'y a obligation de paiement de la part de l'opérateur de réseau que si une centrale photovoltaïque au sol a été construite dans une zone correspondant au plan de développement (STMI, 2009). Cette sanction spatiale du secteur solaire a été considérablement renforcée en 2010, lors de la révision de la loi EEG sur les énergies renouvelables (BGBl. 2010). Il ne s'agit cependant pas d'un arbitraire législatif, puisque les mesures restrictives découlent de la forte baisse des coûts du solaire d'avant 2010 et de l'excessif subventionnement de la branche. Au moment de la révision de la loi, le photovoltaïque représente une part de seulement $12 \%$ du mix électrique (Fig. 3), alors qu'il bénéficie de $40 \%$ (1,5 cents / $\mathrm{kWh})$ de l'ensemble des tarifs d'achat attribués aux renouvelables. Même l'Association fédérale de l'industrie solaire a reconnu que le solaire bénéficiait d'un avantage excessif de la loi EEG et s'est prononcé pour une amélioration fondamentale du dispositif, à travers un découplage de l'offre et de la demande (comme Müller, 2011).

Dans une perspective spatiale, les conséquences de la révision de la loi sont considérables. Un espace vert ayant au cours des trois dernières années été utilisé comme terre cultivée ne peut plus bénéficier du nouveau système de rémunération. Cela doit permettre de stopper la consommation massive d'espace, ainsi que la hausse des prix de location du foncier agricole. De plus, il est important de préserver les bases spatiales d'une agriculture basée sur la production d'aliments et de fourrage. La réforme de la loi montre que la production d'énergie solaire sur surface agricole n'est plus souhaitée (Photo 1). Il en résulte la nécessité pour l'industrie solaire, de mettre désormais l'accent sur les solutions d'intégration au bâti (Schrödter W. / Kuras M., 2011). D'un point de vue écologique, épargner d'anciennes terres arables est d'autant plus souhaitable, que la conversion de terres arables en prairies a d'une part épargné les sols précédemment exploités de façon intensive, et d'autre part, a permis le développement sur ces surfaces (en raison de leur transformation en surfaces de compensation écologique), d'une grande biodiversité floristique et faunistique. Il convient également de noter qu'avec l'exclusion des terres arables et la recommandation d'implanter les projets solaires sur des surfaces en conversion, de nombreuses municipalités ont saisi l'occasion de construire des parcs solaires à proximité des lieux de consommation. Comme on peut trouver de grandes surfaces de terrains en conversion principalement en Allemagne de l'Est, il faut s'attendre également à ce qu'à l'avenir l'essentiel du développement des parcs s'y fasse (Heup J., 2011, p. 52). 
17 Malgré la préférence pour les solutions d'intégration au bâti, dans la loi EEG de 2010 on a proposé des surfaces alternatives aux restrictions concernant les surfaces agricoles. On privilégie les surfaces qui subissent déjà de fortes nuisances. À ce stade, il faut insister sur le fait que cette procédure est en contradiction avec les dispositions de la loi sur l'aménagement du territoire (BMJ, 2008) qui prévoient de créer des conditions de vie égales, puisque dans les régions déjà affectées les impacts spatiaux vont augmenter de manière disproportionnée. Néanmoins, la création d'une installation de plein champ le long d'une infrastructure linéaire est maintenant classée comme pouvant être subventionnée. Le couloir concerné est cependant extrêmement étroit puisqu'il a une largeur de $110 \mathrm{~m}$ à partir du bord extérieur de la ligne de chemin de fer ou de l'autoroute (Journal officiel fédéral, 2010, p. 1171). Le principe posé par les autorités routières des restrictions à la construction dans la plage des 100 mètres et leur interdiction dans la zone des 40 mètres n'exclue plus maintenant aussi catégoriquement la mise en place d'installations de plein champ. La condition pour la levée de l'interdiction de construire est que le bord extérieur de l'autoroute soit très exactement défini dans le plan d'urbanisme, parce que c'est seulement ainsi que les différents types de surfaces peuvent être clairement distingués. Il est aussi important de vérifier si la délimitation de la superficie constructible ne nuit pas à la sécurité et à la fluidité de la circulation. Il faut donc s'assurer qu'aucun reflet nuisible ne provient des installations solaires. Troisièmement, il est nécessaire d'associer le responsable de la construction de la route dans la préparation du plan d'urbanisme. Les restrictions dans la bande des 110 mètres le long des voies de chemin de fer sont sensiblement plus faibles, car dans ce cas, il n'y a aucune interdiction de principe en ce qui concerne la construction. Elle s'applique uniquement à éviter les impacts négatifs sur l'environnement et les éventuels reflets nuisibles (Schrödter W. / Kuras M., 2011).

Ces zones ne seront cependant pas suffisantes pour que le photovoltaïque puisse s'approcher de la contribution nécessaire à la transition énergétique. Par conséquent, dans le contexte de la loi EEG sur les énergies renouvelables, on a créé et élargi une autre catégorie d'espace (la surface en conversion) (BGBl , 2010, p. 1171). À l'origine, la catégorie surface en conversion a été attribuée à des terrains militaires ou relevant de processus de reconversion économique. La construction de parcs photovoltaïques sur des surfaces en conversion anciennement dévolues à l'habitat ou à la circulation donne également droit à bénéficier d'une indemnisation. Une contribution importante de l'énergie solaire au mixe énergétique allemand est toutefois à réaliser principalement sur les terrains militaires. Parmi les exemples, on peut citer l'ancien terrain militaire soviétique de Lieberose (Brandebourg) (First Solar GmbH, 2009), l'ancien aérodrome allemand de Brandis Waldpolenz (Saxe) (SolarServer , 2010) qui étaient à l'origine occupés par l'Armée Rouge, ainsi que et l'ancien aérodrome militaire d'Eberswalde (Brandebourg) (Hybrid solar AG, 2012) , sur lesquels sont installés des centrales photovoltaïques de grandes dimensions atteignant respectivement des capacités de 53 MW (162 ha), 40 MW (110 ha) et 84,5 MW (185 ha). Il est à noter que la construction du parc solaire de Lieberose s'est accompagnée de la dépollution des sols, puisque le développeur du projet, Juwi, s'était engagé à débarrasser le terrain des armes et de la contamination chimique qu'il contenait. Cela montre que la combinaison entre parc solaire et surface dégradée peut apporter une contribution positive à l'environnement, dans la mesure où la remise en état de terrains gravement pollués peut enfin sembler réaliste. Dans ce cas, l'amendement de 2010 à la loi EEG n'est pas contraire à la loi ROG sur l'aménagement du territoire qui prescrit de créer des conditions de vie égales. On a 
pu aussi montrer sur la base d'une étude de suivi du terrain de Lieberose, que les conditions de vie des espèces d'oiseaux menacées s'étaient même améliorées. Les projets dans lesquels des surfaces présentant un important stock de carbone sont détruites constituent une source potentielle de conflit (Heup J., 2011, p. 51). Cela est bien entendu à éviter en matière de protection du climat.

\section{B. Ne pas stopper le photovoltaïque}

Malgré les diminutions de tarifs et les restrictions spatiales réalisées en 2010 dans le domaine du solaire, la forte croissance de la branche n'a pas pu être contenue. Rien que durant la seule année 2011, on a installé 7500 MW de capacité photovoltaïque supplémentaire en Allemagne (Nikionok-Ehrlich A., 2012, p. 21). Au cours de la même période, l'éolien terrestre n'a progressé que de $2085 \mathrm{MW}$ (Weinhold N., 2012, p. 35). Pour 2013, l'expansion du photovoltaïque doit être limitée à 2 500-3500 MW et dans les années suivantes une réduction annuelle de $400 \mathrm{MW}$ doit être initiée. Dans ce but, les ministères de l'économie et de l'environnement ont décidé au printemps 2012 d'ajouter d'autres sanctions à l'industrie solaire. Le compromis consiste à réduire les tarifs d'achat d'un seul coup. En outre, il a été décidé d'appliquer un tarif d'achat dégressif par un abaissement mensuel. Enfin, un maximum de $90 \%$ du courant produit est acheté à un prix garanti ; pour les petites installations c'est même seulement $85 \%$. L'électricité restante doit être consommée sur place, ou commercialisée par le producteur luimême. Enfin, la prime d'autoconsommation est supprimée (BMWi / BMU, 2012). Ces sanctions ont été fortement critiquées par les Länder de l'Est car c'est dans ces régions en particulier que l'industrie solaire était à l'origine de nombreuses créations d'emplois. La Bavière a également exprimé la crainte que l'agriculture, qui avec ses vastes granges et écuries, possède un énorme potentiel pour l'installation du photovoltaïque, puisse perdre une source alternative de revenus (Nikionok-Ehrlich A., 2012).

Les scientifiques de l'Institut RWTH (Institut rhéno-westphalien de recherche économique) attribuent la hausse des coûts de la transition énergétique à l'expansion massive du photovoltaïque découlant de l'inefficacité de l'économie planifiée créée par la loi EEG et se prononcent pour la mise en place d'un modèle de quotas conforme au marché (Frondel M. et al., 2011). Cette approche purement économique qui exclut tous les aspects environnementaux et sociétaux, et donc laisse de côté la nécessaire perspective systémique, manque cependant de clairvoyance. D'une part, le modèle des quotas ne pourrait pas garantir une sécurité de planification; d'autre part, les avantages de coûts à long terme des technologies, à partir du moment où on atteint une production de masse et une standardisation, ne sont pas pris en considération. Plus généralement cette approche ne tient pas compte du fait que les technologies renouvelables se trouvent à différents stades du cycle de vie des produits; ce qui rend difficile une comparaison. 


\section{La stabilisation des réseaux électriques}

\section{A. Le changement de système énergétique recèle des dangers}

21 Afin d'assurer la sécurité du fonctionnement du réseau électrique et d'éviter ainsi une coupure de courant, il est nécessaire d'ajuster la production et la consommation d'électricité. L'indicateur de stabilité du réseau est la fréquence, qui varie autour de la valeur nominale de $50 \mathrm{~Hz}$ (Hermann M., 2012). En raison de la fiabilité du système basé sur les énergies fossiles et nucléaires, les fluctuations de la production électriques étaient jusqu'à présent l'exception. Seules les pannes de centrales ont pu conduire à des fluctuations imprévisibles dans le fonctionnement du réseau ; mais elles étaient faciles à maîtriser grâce à l'utilisation de centrales de pointe flexibles, rapides à mettre en marche. Avec le passage aux énergies renouvelables cette stabilité du système d'exploitation du réseau ne va plus de soi. Au lieu de cela, la prévisibilité de l'approvisionnement électrique diminuera avec l'expansion des systèmes éoliens et solaires, car les apports attendus de courant électrique vont connaître une forte variabilité temporelle et spatiale, en raison de fluctuations à court terme (en fonction du temps), à moyen terme (saisonnières) et à long terme (liées au climat). La stabilisation des réseaux va s'avérer d'autant plus difficile qu'un système d'énergies renouvelables n'est pas composé de quelques producteurs de courant contrôlés de manière centralisée, mais d'innombrables fournisseurs décentralisés d'électricité renouvelable.

\section{B. Les centrales à cycle combiné}

22 Du point de vue géographique, il y a déjà des approches prometteuses afin de pouvoir maintenir l'exploitation du réseau malgré la complexité d'un approvisionnement complet à partir d'énergies renouvelables. La recherche en météorologie de l'énergie s'est par exemple fixée pour tâche de mettre à disposition des gestionnaires de réseaux des informations météorologiques avec une résolution spatiale élevée, afin de pouvoir prédire les contributions attendues des sources d'énergie intermittentes. À partir de prévisions météorologiques numériques réalisées au moyen de régressions statistiques, on peut prévoir la production des éoliennes et des fermes solaires sur un à trois jours. Grâce à l'analyse des données satellitaires et à l'extrapolation des structures nuageuses, il est également possible de préciser la production des centrales solaires dans un délai de quelques heures (Heinemann D., 2006 ; Luther J., 2006).

Cette assistance de la météorologie de l'énergie pourrait apporter une contribution importante à une combinaison intelligente de sources d'énergie renouvelables sous la forme de centrales à cycle combiné. Les avantages spécifiques des différentes technologies peuvent être ainsi utilisés pour compenser les inconvénients des autres (Bosch S. / Peyke G., 2010; Mackensen R. et al., 2008). La combinaison dans l'espace de l'énergie éolienne et de l'énergie solaire offre la possibilité de produire du courant électrique à partir de sources renouvelables, tant en période de basse pression que de haute pression. Plus on augmente l'espace au sein duquel les éoliennes et les centrales solaires sont associées et plus on accroit la probabilité que l'énergie éolienne et l'énergie solaire soient en mesure d'alimenter en même temps le réseau. Dans le contexte d'un réseau européen, les potentiels énergétiques régionaux et nationaux 
spécifiques pourrait ainsi se compléter. Les divers phénomènes météorologiques régionaux et nationaux pourraient se compenser et donc augmenter la stabilité du réseau (Popp M., 2010, p. 56). Des problèmes ne pourraient alors se produire que si, par exemple lors d'une période de vents faibles pendant les mois d'hiver, ni le vent, ni l'énergie solaire ne seraient alors disponibles en quantités suffisantes. Cependant, ce manque de courant pourrait être compensé par du courant produit à partir de la biomasse, car les installations de biogaz offrent la possibilité de stocker le biogaz brut, ainsi que le biométhane obtenu par transformations successives. L'énergie de la biomasse peut ainsi être mise à disposition avec un décalage spatial et temporel en fonction des besoins. En plus de la biomasse, l'énergie géothermique pourrait également servir en tant que source d'énergie de charge de base. À cet égard, il existe en Allemagne un grand potentiel énergétique à peine exploité (Bundesverband Geothermie, 2011).

La combinaison du biogaz, des éoliennes et des centrales solaires offrirait la possibilité de fournir de grandes quantités d'électricité renouvelable indépendamment des conditions météorologiques. Ajouter à cela une centrale à cycle combiné et une centrale hydroélectrique à accumulation par pompage, on pourrait même disposer d'un excédent de courant électrique (Mackensen R. et al, 2008 ; Wiedemann K., 2011a). Le problème cependant est que la capacité de ces centrales de pompage-turbinage, qui seraient actuellement nécessaires au marché allemand de l'électricité, n'est que de 0,04 TWh alors qu'il n'existe pratiquement aucun potentiel supplémentaire à construire dans la partie montagneuse du pays (Janzing B., 2010, p. 29; Popp M., 2010, p. 57). Même en prenant en compte le stockage de l'air comprimé dans des cavernes et des cavités souterraines, on ne pourrait pas disposer de la capacité de stockage d'environ $40 \mathrm{TWh}$ qui serait nécessaire à long terme (Sedlacek R., 2009, p. 412). C'est pourquoi on a expressément besoin de solutions alternatives de stockage ; sinon il sera nécessaire de développer une connexion aux centrales de pompage-turbinage scandinaves.

\section{Les synergies spatiales entre l'énergie éolienne et la biomasse}

Pendant ce temps, de grands espoirs sont mis dans ce que l'on appelle la technologie de power to gas dont il n'existe que des installations-pilotes (Hüttenrauch J. / MüllerSyring G., 2010; Janzing B., 2011; Rieke P., 2011; Wächter F., 2012; Wiedemann K., 2011b; Petersen N.H., 2012). Cette technologie est destinée à permettre de stocker les surplus d'électricité renouvelable afin de pouvoir les restituer au réseau lorsqu'il y a des besoins à certains moments ou dans certains espaces. On parvient à ce résultat en combinant l'électrolyse, la méthanisation et le gaz utilisé dans les centrales de cogénération. Dans un premier temps, on utilise l'énergie éolienne excédentaire pour décomposer l'eau en hydrogène et en oxygène (électrolyse). L'hydrogène peut être stocké et converti plus tard en électricité et en chaleur en utilisant des piles à combustible. En outre, il est possible d'alimenter le réseau de gaz naturel avec de l'hydrogène, dont la part selon la loi, ne doit pas dépasser $5 \%$, afin de ne pas endommager les turbines à gaz et autres appareils sensibles (Valentin F. / von Bredow H., 2011, p. 102). La méthanisation des excédents d'énergie éolienne permet une meilleure utilisation de l'infrastructure existante de gazoducs. Il s'agit d'utiliser une réaction entre l'hydrogène et le dioxyde de carbone (réaction Sabatier) pour créer ce que l'on appelle le méthane renouvelable, un dispositif de stockage d'énergie, qui peut être facilement intégré dans les gazoducs et les réservoirs de stockage de gaz existants. 
Cela permet de disposer d'une capacité de stockage de 220 TWh, soit suffisamment pour approvisionner l'Allemagne en électricité pendant trois mois. Avec une liaison bidirectionnelle entre le réseau électrique et le réseau de gaz on peut aussi en cas de besoin, transformer le biométhane en électricité. Pour cela, on a besoin d'une turbine à gaz ou d'une centrale de cogénération. L'avantage d'un découplage temporel et spatial de la production et de la consommation d'énergie est compensé par l'inconvénient d'un faible rendement. Si la production d'électricité n'est pas réalisée par production combinée de chaleur et d'électricité, le rendement est réduit à 35\% (Lubbadeh J. / Honselmann G., 2013, p. 70). Pour cette raison, l'adoption de cette technologie est dépendante de la réalisation d'autres améliorations.

Une optimisation spatiale pourrait résulter de la proximité des sites de production de biogaz et des éoliennes, parce que les installations de biogaz qui sont utilisées pour la production de biométhane par séparation du dioxyde de carbone pourraient servir de source de carbone pour la réaction Sabatier. Le potentiel de dioxyde de carbone issu de la production de biogaz serait quoi qu'il en soit suffisant pour satisfaire la demande en CO2 pour le méthane renouvelable dans le cas d'un approvisionnement complet avec des énergies renouvelables (Trost T. et al., 2012, p. 172). Les éoliennes fournissent ainsi l'énergie nécessaire au fonctionnement des électrolyseurs, puisque l'hydrogène produit réagit avec le dioxyde de carbone dégagé par les installations de biogaz. Cependant, comme la mise sur le marché des installations de méthane renouvelable n'est pas attendue avant 2040 (Nitsch J. et al., 2010, p. 78), les projets d'extension des réseaux d'électricité ne doivent plus être davantage différés.

\section{Les incertitudes concernant le renforcement du réseau}

Actuellement, il est nécessaire de renforcer les réseaux d'électricité sur 1700 à $3600 \mathrm{~km}$ et de modifier les lignes existantes sur une longueur totale qui pourrait atteindre 5700 km (DENA, 2012, p. 13; Petersen N.H., 2012; Wächter F., 2012) (figure 4). Force est de constater que jusqu'à présent, seuls $100 \mathrm{~km}$ ont été réalisés sur les $850 \mathrm{~km}$ de lignes à haute tension jugés nécessaires. Les goulots d'étranglement sur le réseau sont principalement entre l'Ouest et l'Est de l'Allemagne. Cette situation est un héritage de la division politique et économique qui existait au temps de la guerre froide (Zimmermann J.-R. / Weinhold N., 2011, p. 17). Par conséquent, le développement des énergies renouvelables donne en même temps l'occasion de mieux relier l'Est et l'Ouest de l'Allemagne. 
Figure 4 : mixes énergétiques des différents Länder et nouvelles lignes électriques prévues en Allemagne

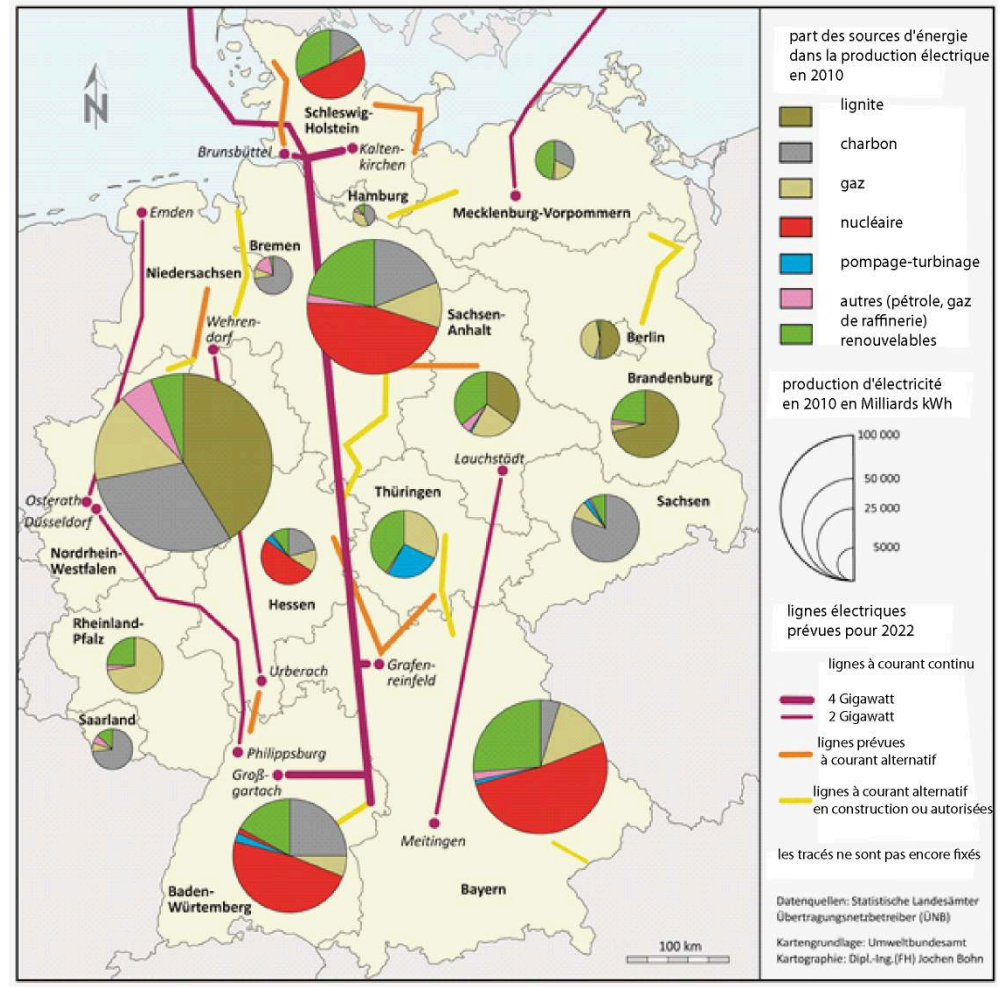

En ce qui concerne la question de l'étendue réelle de la reconfiguration du réseau à réaliser, les avis sont assez divergents. Ceci s'explique par le fait qu'on ne sait pas encore si le futur système d'énergie renouvelable aura un caractère plutôt décentralisé, ou s'il reposera sur quelques grands projets à commande centralisée -tels que Desertec (Walter K. / Bosch S., 2013.), ou les grands parcs éoliens offshore (Bosch S. / Peyke G., 2011a). Quoi qu'il en soit, il faut noter qu'il ne s'agit pas seulement d'une reconfiguration à l'échelle nationale, mais dans le cadre d'un réseau européen d'interconnexion. À cet effet, il serait donc opportun d'harmoniser les instruments de financement, ainsi que les prix de l'électricité, car c'est seulement à cette condition qu'il sera possible de connecter par exemple, un parc éolien allemand au réseau néerlandais. Dans le cadre d'une extension du réseau transnational, les États qui se voient actuellement confrontés à des prix élevés du courant électrique, pourraient avoir accès à des prix plus bas de l'électricité. Les consommateurs norvégiens par contre, qui sont habitués à des prix bas de l'électricité, devraient s'attendre à des augmentations de prix. Dans ce contexte, il est compréhensible que la construction de la nouvelle ligne électrique entre l'Allemagne et la Norvège NorGer, puisse être retardée. En revanche, le développement des réseaux entre l'Irlande et le Pays de Galles (Eir Grid Eastwest-Interconnector), entre la Grande- Bretagne et la Belgique (Nemo), entre la Suède et la Finlande (Fenno-Skan2), entre l'Estonie et la Finlande (Eastlink 2) et entre les Pays-Bas et le Danemark (Cobra Cable) sont accélérés (Zimmermann J.-R. / Weinhold N., 2011, p. 17). L'Europe se consolide au moins au niveau du marché de l'électricité. 


\section{Conclusion : énergies renouvelables et géographie, la perspective systémique}

La mise à disposition des surfaces est sans doute le talon d'Achille de la transition énergétique en Allemagne, car les énergies renouvelables doivent être intégrées dans un espace qui est déjà largement utilisé par des utilisations concurrentes des terres. De plus, en raison de la progression constante des sources d'énergie intermittentes, il devient de plus en plus difficile de stabiliser le réseau. À cet égard, l'article a montré que les perspectives des sciences spatiales sont susceptibles de donner un nouvel élan à une expansion intelligente des technologies des renouvelables. La diminution de l'acceptation de la production décentralisée peut être interprétée comme une preuve qu'il ne suffit pas de laisser le développement des énergies renouvelables aux seules sciences de l'économie et de l'ingénierie, et donc de placer la révolution énergétique sous le joug technocratique. Sans la prise en compte de l'espace comme ressource, la perspective systémique nécessaire pour réduire le nombre de conflits d'utilisation des terres, doit être abandonnée. Avec le retour de «l'energy from space», la géographie retrouve une place essentielle dans le complexe des recherches sur l'énergie en général. Cependant, elle ne peut assumer cette responsabilité qu'en s'appuyant sur sa plus grande force - le point de vue systémique - en agissant comme un intermédiaire entre les hommes politiques, les économistes, les ingénieurs, les architectes paysagistes et les écologistes. Les géographes ne réfléchissent pas seulement au meilleur emplacement d'une centrale d'énergie renouvelable, mais leur intérêt se porte aussi, au-delà de la localisation de la centrale elle-même, sur les effets de son intégration technologique. Le développement des énergies renouvelables est toujours à réévaluer dans le contexte des différentes conditions locales.

\section{BIBLIOGRAPHY}

Bayerische Staatsministerien (StMI)(StMWFK)(StMF)(StMWIVT)(StMUG)(StMELF), 2011, Hinweise zur Planung von Windkraftanlagen (WKA). München.

Bayerisches Staatsministerium des Innern (StMI), 2009, Freiflächen-Photovoltaikanlagen. http:// www.innenministerium.bayern.de/imperia/md/content/stmi/bau-en/

rechtundtechnikundbauplanung/_baurecht/rundschreiben/photovoltaik.pdf (15.12.2010).

Boeing N., 2011, „Mehr Watt, bitte!“ In: Technology Review - Das M.I.T.-Magazin für Innovation (1), Spezialheft Energie, S. 30-31.

Bosch S., 2012, Erfassung und Bewertung des Einflusses der Ressource Raum im Rahmen der Förderung von Erneuerbaren Energien sowie Ableitung eines ganzheitlichen Ansatzes zur optimalen Integration von regenerativen Technologien in den ländlichen Raum. Augsburg.

Bosch S., 2013, „Erneuerbare Energie für Deutschland - Räumliche und technische Planung für eine intelligente Energieversorgung“. In: Geographische Rundschau (1), p. 4-11. 
Bosch S., Peyke G., 2009, „Energiewende durch GIS“. In: GIS.Business (8), p. 44-46.

Bosch S., Peyke G. 2010, „Raum und Erneuerbare Energien - Anforderungen eines regenerativen Energiesystems an die Standortplanung“. In: Standort - Zeitschrift für Angewandte Geographie (1), p. 11-19.

Bosch S., Peyke G.,2011a, „Erneuerbare Energien und Offshore-Standorte. Rückzug oder Zukunftsperspektive?“ In: Geographische Rundschau (4), p. 51-59.

Bosch S., Peyke G., 2011b, „Gegenwind für die Erneuerbaren - Räumliche Neuorientierung der Wind-, Solar- und Bioenergie vor dem Hintergrund einer verringerten Akzeptanz sowie zunehmender Flächennutzungskonflikte im ländlichen Raum“. In: Raumforschung und Raumordnung (2), p. 105-118.

Bosch S., Peyke G., 2011c, Regionalplanerische Einstufung der Wind-energie in Deutschland Visualisierung konkurrierender Flächennutzungsansprüche an On- und Offshore-Standorten mittels GIS. In: Strobl, J., Blaschke, T., Griesebner, G. (Hrsg.), Angewandte Geoinformatik 2010 Beiträge zum 23. AGIT-Symposium Salzburg, p. 450-459.

Bosch S., Peyke G., 2011d, , Kein Raum für die Erneuerbaren? GIS hilft bei der Suche! “ In: GIS.Business - Zeitschrift für Geoinformation (5), p. 34-36.

Brand C., Pöhlmann K., 2010, „Konzentrationsplanung von Windkraftanlagen durch Flächennutzungsplan - Wo beginnt die Verhinderungsplanung?“ Zum Urteil des BVerwG v. 20.05.2010 - 4 C 7.09. In: Zeitschrift für Neues Energierecht (5), p. 476-479.

Brücher W., 2008, „Erneuerbare Energien in der globalen Versorgung aus historischgeographischer Perspektive“. In: Geographische Rundschau (1), p. 4-12.

Bundesgesetzblatt (BGBl), 2010, Erstes Gesetz zur Änderung des Erneuerbare-Energien-Gesetzes http://www.bgbl.de/Xaver/start.xav?

startbk=Bundesanzeiger_BGBl\&bk=Bundesanzeiger_BGBl\&start=//*

[@attr_id=\%27bgbl110s1170.pdf\%27]; consulté le 28.09.2012.

Bundesinstitut für Bau-, Stadt- und Raumforschung (BBSR), 2008, Raumordnungsplan-Monitor, http://www.bbsr.bund.de/nn_21918/BBSR/DE/Raumentwicklung/RaumentwicklungDeutschland/LandesRegionalplanung/Projekte/Roplamo/roplamo.html, (20.8.2012).

Bundesministerium für Justiz (BMJ), 2008, Raumordnungsgesetz (ROG). http://www.gesetze-iminternet.de/bundesrecht/rog_2008/gesamt.pdf (24.09.2013).

Bundesministerium für Wirtschaft und Technologie (BMWi) und Bundesministerium für Umwelt, Naturschutz und Reaktorsicherheit (BMU), 2012, EU-Effizienzrichtlinie und ErneuerbareEnergien-Gesetz. Ergebnispapier. Berlin.

Bundesverband der Energie- und Wasserwirtschaft (BDEW): Anteil der Erneuerbaren Energien steigt auf 23 Prozent - Pressemitteilung vom 18.12.2012, http://www.bdew.de/internet.nsf/id/ 20121218-pi-anteil-der-erneuerbaren-energien-steigt-auf-23-prozent-de (24.04.2013).

Bundesverband Geothermie, 2011, Nutzung der Geothermie in Deutschland, www.geothermie.de/ wissenswelt/geothermie/in-deutschland.html (15.03.2012).

Bundesverband Windenergie (BWE): Statistiken - Windenergie in Bayern. http://www.windenergie.de/infocenter/statistiken/bundeslaender/windenergie-bayern (24.09.2013).

Bund Naturschutz, 2010, Kommunen als Klimaschützer gefragt - Vorbildhafte Energieversorgung in der Gemeinde Wildpoldsried, http://www.bund-naturschutz.de/presse/pressemitteilungen/ detail/artikel/1602/pm/77b7c4b7aff012ad8ea3fofc6da95d5e.html (26.9.2012). 
Deutsche Energie-Agentur (dena) (Hrsg.), 2010, dena-Netzstudie II. Integration erneuerbarer Energien in die deutsche Stromversorgung im Zeitraum 2015-2010 mit Ausblick 2025. Berlin.

Einig, K., Heilmann, J., Zaspel, B., 2011, Wie viel Platz die Windkraft braucht. In: Neue Energie (8), p. 34-37.

First Solar GmbH, 2009, Größtes Solarkraftwerk in Deutschland errichtet, http://www.solarparklieberose.de/ (27.9.2012).

Fraunhofer Institut für Windenergie und Energiesystemtechnik (IWES), 2011, (Hrsg.), Studie zum Potenzial der Windenergienutzung an Land. Kassel.

Frondel M., Schmidt C. M., aus dem Moore N., 2011, „Explodierende Kosten: Auswirkungen der Photovoltaikförderung in Deutschland“. In: BWK - Das Energie-Fachmagazin (3), p. 136-139.

Gailing L., Leibenath M. (Hrsg.), 2013, Neue Energielandschaften - Neue Perspektiven der Landschaftsforschung. Heidelberg.

Hasse J., 1999, Bildstörung - Windenergie und Landschaftsästhetik, Wahrnehmungsgeographische Studien zur Regionalentwicklung, Oldenburg.

Heinemann D., 2006, Energiemeteorologie: Ein Überblick. In: Heinemann, D./ Hoyer-Klick, C. (Hrsg.): Energiemeteorologie, Work-shop, 2. November 2006, Berlin, p. 16-21.

Henkel G., 2004, Der Ländliche Raum - Gegenwart und Wandlungsprozesse seit dem 19. Jahrhundert in Deutschland. Berlin, Stuttgart.

Hermann M., 2012, „Regelenergie ergrünt“. In: Erneuerbare Energien (1), p. 20-23.

Heup J., 2011, „Aufruhr auf der Freifläche“. In: Neue Energie (12), p. 50-53.

Hüttenrauch J., Müller-Syring G., 2010, „Zumischung von Wasserstoff zum Erdgas“. In: Energie wasserpraxis (10), p. 68-71.

Janzing B., 2010, „Kraft auf Vorrat“. In: Neue Energie (7), p. 24-31.

Janzing B., 2011, „Windgas für jedermann“. In: Neue Energie (7), p. 35-37.

Karl F., 2006, Erneuerbare Energien als Gegenstand von Festlegungen in Raumordnungsplänen. In: Akademie für Raumforschung und Landesplanung (Hrsg.), Arbeitsmaterialien 319. Hannover.

Klagge B. \& Brocke T., 2013, „Energiewende vor Ort. Dezentrale Stromerzeugung und die Rolle von Stadtwerken und Regionalversorgern“. In: Geographische Rundschau (1), p. 12-18.

Knies J., 2010, „Windkraftanlagen in Schottland - Möglichkeiten von Sichtbarkeitsanalysen“. In: Strobl J., Blaschke T., Griesebner G. (Hrsg.), Angewandte Geoinformatik 2010 - Beiträge zum 23. AGIT-Symposium Salzburg, p. 514-522.

Leibenath M. \& Otto A., 2012, „Diskursive Konstituierung von Kulturlandschaft am Beispiel politischer Windenergiediskurse in Deutschland“. In: Raumforschung und Raumordnung (2), p. 119-131.

Leidorf K., 2012, Luftbilddokumentation. www.leidorf.de/index.php? option=com_frontpage \&Itemid=1 (03.05.2012).

Lubbadeh J, Honsel G., 2013, „Was spricht für, was gegen Windgas?“ In: Technology Review Magazin für Innovation, Spezialheft Energie, p. 70-71.

Luther J., 2006, Wege zu einer nachhaltigen Energieversorgung - die Bedeutung der Energiemeteorologie, in: Heinemann, D./ Hoyer-Klick, C. (Hrsg.), Energiemeteorologie, Workshop, 2. November 2006, Berlin. p. 6-13. 
Mackensen R., Schlögl F., Rohrig K., Adzic L., Saint-Drenan Y. M., 2008, Das regenerative Kombikraftwerk, Kassel, http://www.iset.uni-kassel.de/abt/FB-I/publication/ 2008-001_Das_regenerative_Kombikraftwerk_Paper.pdf (18.5.2012).

Müller B., 2011, „Verflixt und zugepflastert“. In: Technology Review (6), p. 62-67.

Nikionok-Ehrlich A., 2012, „Sein oder Nicht-Sein“. In: Neue Energie (2), p. 20-22.

Nitsch J., Pregger T., Scholz Y., Naegler T., Sterner M., Gerhardt N., Oehsen A. v., Pape C., SaintDrenan Y. M., Wenzel B., 2010, Leitstudie 2010 - Langfristszenarien und Strategien für den Ausbau der erneuerbaren Energien in Deutschland bei Berücksichtigung der Entwicklung in Europa und global. Stuttgart.

Nohl W., 2001, „Ästhetisches Erlebnis von Windkraftanlagen in der Landschaft“, Naturschutz und Landschaftsplanung, Bd. 33, (12), p. 365-372.

Nohl W., 2010, „Landschaftsästhetische Auswirkungen von Windkraft-anlagen“. In: Bayerischer Landesverein für Heimatpflege e.V. (Hrsg.): Schönere Heimat - Erbe und Auftrag (1), p. 3-12.

Petersen N. H., 2012, „Run auf den Speicher“. In: Erneuerbare Energien (3), p. 16-19.

Peters J., 2010, Landschaft als Energieressource - Biomasseproduktion und die Auswirkungen auf die Kulturlandschaft. Fachtagung „Raumplanung und die steigende Nutzung von Bioenergie, am 28. September 2010 Wetzlar, http://www.na-hessen.de/downloads/ 10n102biomassekulturlandschaft.pdf (15.12.2011).

Peyke G., Bosch S., Färber K., Tatu D., Walter K., 2013, Erneuerbare Energien von A bis Z. In: Friedmann A., Hilpert M., Jacobeit J., Kunstmann H., Ohl U., Peyke G., Reller A., Schneider T., Thieme K., Timpf S., Wetzel K.-F. (Hrsg.), Geographica Augustana, Bd. 13, Augsburg.

Popp M., 2010, „Regenerativstrom im Ringwall speichern“. In: BWK - Das Energie-Fachmagazin (12), p. 53-58.

Rempel H., 2008, „, Globale Verfügbarkeit nicht-erneuerbarer Energierohstoffe“. In: Geographische Rundschau (1), p. 22-31.

Rieke S., 2011, „Windstrom wird in Methan gespeichert“. In: Biogas Journal (1), p. 89-93.

Scheidler A., 2010, „Verunstaltung des Landschaftsbildes durch Wind-kraftanlagen“. In: Natur und Recht (8), p. 525-530.

Schmitt T., 2012, „Postfordistische Energiepolitiken? Das Desertec-Konzept als Szenario zur Restrukturierung der Energieversorgung in der EUMENA-Region“. In: Zeitschrift für Wirtschaftsgeographie (4), p. 244-263.

Schöbel S., 2012, Windenergie und Landschaftsästhetik. Zur landschaftsgerechten Anordnung von Windfarmen. Berlin.

Schrödter W. \& Kuras M., 2011, „Auswirkungen des EEG 2010 auf die Planung von Flächen für Photovoltaikanlagen“. In: Zeitschrift für Neues Energierecht (2), p. 144-151.

Sedlacek R., 2009, „Untertage-Gasspeicherung in Deutschland“. In: Erdöl Erdgas Kohle (11), p. 412-426.

Solarhybrid AG, 2012, FinowTower I+II, http://www.solarhybrid.ag/Die-Entstehung-desgroessten-Solarkraftwerks-Europas.ref_finowtowerii.0.html (27.9.2012).

SolarServer, 2010, Energiepark Waldpolenz ist größtes Solar-Kraftwerk der Welt, http:// www.solarserver.de/news/news-9035.html (27.9.2012). 
Trost T., Jentsch M., Holzhammer U., Horn S., 2012, Die Biogasanlagen als zukünftige CO2Produzenten für die Herstellung von erneuerbarem Methan - Power-to-Gas als Langzeitspeicher und alternativer Kraftstoff. In: gwf-Gas/ Erdgas (3), p. 172-179.

Valentin F. \& v. Bredow H., 2011, „Power-to-Gas: Rechtlicher Rahmen für Wasserstoff und synthetisches Gas aus erneuerbaren Energien“. In: Energiewirtschaftliche Tagesfragen (12), p. 99-105.

Wächter F., 2012, „Strom zu Erdgas“. In: Erneuerbare Energien (3), p. 22-25.

Walter K. \& Bosch S., 2013, "Intercontinental cross-linking of power supply - calculating an optimal power line corridor from North Africa to Central Europe". In: Energy, Sustainability and Society. DOI: 10.1186/2192-0567-3-14.

Weinhold N., 2012, “Der Süden dreht auf”. In: Neue Energie (2), p. 35-39.

Wiedemann K., 2011a, „Einspeisen auf Knopfdruck“. In: Neue Energie (6), p. 58-60.

Wiedemann K., 2011b, „Der unsichtbare Speicher“. In: Neue Energie (7), p. 30-34.

Wikipedia, 2012, Eintrag „Ölfeld“, http://de.wikipedia.org/wiki/\%C3\%96lfeld (25.9.2012).

World Nuclear Association (WNA) (2013): World Nuclear Power Reactors \& Uranium Requirements. http://world-nuclear.org/info/Facts-and-Figures/World-Nuclear-PowerReactors-and-Uranium-Requirements/\#.UkKPfD16FnU (25.09.2013).

Zimmermann J.-R., 2011a, „50 Prozent plus - Strom aus Landwind“. In: Neue Energie (4), p. 37-42. Zimmermann J.-R., 2011b, „Im Windschatten“. In: Neue Energie (10), p. 40-42.

Zimmermann, J.-R., 2012, „Mehr Mühlen erbeten“. In: Neue Energie (2), p. 24-25.

Zimmermann J.-R. \& Weinhold N., 2011, „Das Netz wächst zusammen“. In: Neue Energie (12), p. 17-23.

\section{ABSTRACTS}

The transformation of the energy system in Germany has increased pressure on the different uses of rural areas, so that any further expansion of renewable energy appears as problematic. This requires a balance between energy use and space costs they generate. This is certainly a major challenge for the greatest share of renewable energy development is yet to come.

La transformation du système énergétique en Allemagne a accentué la pression sur les différents usages de l'espace rural, de telle sorte que toute extension supplémentaire des énergies renouvelables apparaît comme problématique. Cela nécessite un rééquilibrage entre les usages énergétiques et les coûts spatiaux qu'ils génèrent. C'est en tout cas un défi majeur car la plus grande part du développement des énergies renouvelables est encore à venir.

Die Umgestaltung des Energiesystems in Deutschland hat den Nutzungsdruck auf den ländlichen Raum in einer Weise erhöht, die jeglichen weiteren Ausbau als problematisch erscheinen lässt und nach einer sensiblen Abwägung zwischen energetischem Nutzen und räumlichen Kosten verlangt. Dies dürfte sich als eine große Herausforderung darstellen, denn der größte Teil des Ausbaus steht Deutschland noch bevor. 
INDEX

Schlüsselwörter: erneuerbare Energien, Flächennutzungskonflikte, Landschaftsästhetik, Netzausbau, räumliche Ressourcen

Keywords: landscape aesthetics, renewable energy, spatial resources, strengthening the power grid, use conflicts

Mots-clés: conflits d'usage, énergie renouvelable, esthétique paysagère, renforcement du réseau, ressources spatiales

\section{AUTHOR}

\section{STEPHAN BOSCH}

Universität Augsburg - Stephan.bosch@geo.uni-augsburg.de 